ARTICLE

\title{
The nucleotide pGpp acts as a third alarmone in Bacillus, with functions distinct from those of (p)ppGpp
}

\author{
Jin Yang (1) 1, Brent W. Anderson (1) 1, Asan Turdiev², Husan Turdiev², David M. Stevenson', \\ Daniel Amador-Noguez (D) ${ }^{1}$, Vincent T. Lee (1) ${ }^{2 凶}$ \& Jue D. Wang (iD) ${ }^{1 凶}$
}

The alarmone nucleotides guanosine tetraphosphate and pentaphosphate, commonly referred to as (p)ppGpp, regulate bacterial responses to nutritional and other stresses. There is evidence for potential existence of a third alarmone, guanosine-5'-monophosphate-3'diphosphate ( $\mathrm{pGpp}$ ), with less-clear functions. Here, we demonstrate the presence of $p G p p$ in bacterial cells, and perform a comprehensive screening to identify proteins that interact respectively with pGpp, ppGpp and pppGpp in Bacillus species. Both ppGpp and pppGpp interact with proteins involved in inhibition of purine nucleotide biosynthesis and with GTPases that control ribosome assembly or activity. By contrast, pGpp interacts with purine biosynthesis proteins but not with the GTPases. In addition, we show that hydrolase NahA (also known as Yvcl) efficiently produces pGpp by hydrolyzing (p)ppGpp, thus modulating alarmone composition and function. Deletion of nahA leads to reduction of $\mathrm{pGpp}$ levels, increased ( $\mathrm{p}$ )ppGpp levels, slower growth recovery from nutrient downshift, and loss of competitive fitness. Our results support the existence and physiological relevance of $p G p p$ as a third alarmone, with functions that can be distinct from those of ( $p$ )ppGpp.

\footnotetext{
${ }^{1}$ Department of Bacteriology, University of Wisconsin, Madison, WI 53706, USA. ${ }^{2}$ Department of Cell Biology and Molecular Genetics, University of Maryland, College Park, MD, USA. ${ }^{\circledR}$ email: vtlee@umd.edu; wang@bact.wisc.edu
} 
O rganisms from bacteria to humans rely on timely and appropriate responses to survive various environmental challenges. The stress signaling nucleotides guanosine tetraphosphate (ppGpp) and guanosine pentaphosphate (pppGpp) are conserved across bacterial species. When induced upon starvation and other stresses, they mediate multiple regulations and pathogenesis by dramatically remodeling the transcriptome, proteome, and metabolome of bacteria in a rapid and consistent manner ${ }^{1-3}$. (p)ppGpp interacts with diverse targets including RNA polymerases in Escherichia coli $^{4-8}$, replication enzyme primase in Bacillus subtilis ${ }^{9-11}$, purine nucleotide biosynthesis enzymes ${ }^{12-15}$, and GTPases involved in ribosome assembly ${ }^{16-19}$. Identification of (p)ppGpp-binding targets on a proteome-wide scale is one way to unravel a more extensive regulatory network ${ }^{15,18,20}$. However, because binding targets differ between different species and most interactomes have not been characterized, the conserved and diversifying features of these interactomes remain incompletely understood.

Another understudied aspect of (p)ppGpp regulation is whether ppGpp and pppGpp, while commonly referred to and characterized as a single species, target the same or different cellular pathways ${ }^{21}$. In addition, there is evidence for potential existence of a third alarmone, guanosine-5'-monophosphate- $3^{\prime}$ diphosphate (pGpp), since several small alarmone synthetases can synthesize $\mathrm{pGpp}$ in vitro ${ }^{22,23}$. However, the clear demonstration of pGpp in bacterial cells has been challenging. More importantly, the regulation specificities and physiological importance of having multiple closely related alarmones in bacteria have not been systematically investigated.

Here we demonstrate pGpp as a third alarmone in Grampositive bacteria by establishing its presence in cells, systematically identifying its interacting targets, and revealing a key enzyme for pGpp production through hydrolyzing (p)ppGpp. We also compare the targets of pGpp, ppGpp, and pppGpp through proteomic screens in Bacillus anthracis. We found that both pppGpp and ppGpp regulate two major cellular pathways: purine synthesis and ribosome biogenesis. In contrast, pGpp strongly regulates purine synthesis targets but does not regulate ribosome biogenesis targets, indicating a separation of regulatory function for these alarmones. In B. subtilis and B. anthracis, pGpp is efficiently produced from pppGpp and ppGpp by the Nucleoside Diphosphate linked to any moiety "X" (NuDiX) NuDiX alarmone hydrolase A (hydrolase NahA), both in vitro and in vivo. A $\Delta n a h A$ mutant has significantly stronger accumulation of pppGpp and decreased accumulation of pGpp, as well as slower recovery from stationary phase and reduced competitive fitness against wild-type cells. Our work suggests a mechanism for the conversion and fine tuning of alarmone regulation and the physiological production of the alarmone pGpp.

\section{Results}

Proteome-wide screen for binding targets of pppGpp and ppGpp from Bacillus anthracis. To systematically characterize the binding targets of (p)ppGpp and identify novel (p)ppGppbinding proteins in Bacillus species, we screened an open reading frame (ORF) library of 5341 ORFs from the pathogen Bacillus anthracis (Fig. 1a). Using Gateway cloning, we placed each ORF into two expression constructs, one expressing the ORF with an $\mathrm{N}$-terminal histidine (His) tag and the other with an $\mathrm{N}$-terminal histidine maltose binding protein (HisMBP) tag.

We first characterized the binding targets of ppGpp using the B. anthracis library. To this end, each ORF in the HisMBP-tagged library was overexpressed and binding to $\left[5^{\prime}-\alpha-{ }^{32} \mathrm{P}\right]-\mathrm{ppGpp}$ was assayed using differential radial capillary action of ligand assay (DRaCALA) ${ }^{24}$ (Fig. 1a). The fraction of ligand bound to protein in each lysate was normalized as a $Z$-score of each plate to reduce the influence of plate-to-plate variation (Supplementary Data 1). We found that the strongest ppGpp-binding targets in $B$. anthracis can be categorized to three groups: (1) purine nucleotide synthesis proteins (Hpt1, Xpt, Gmk, GuaC, PurA, and PurR); (2) ribosome and translation regulatory GTPases (HflX, Der, Obg, RbgA, TrmE, and Era); and (3) nucleotide hydrolytic enzymes, including NuDiX hydrolases and nucleotidases (Fig. 1b). We compared these targets to those obtained from previous screens for ppGpp targets in $E$. coli and for an unseparated mix of pppGpp and ppGpp in $S$. aureus ${ }^{18}$. Comparison of our results with these previous screens yielded conserved themes (Fig. 1b). Among the most conserved themes are the purine nucleotide synthesis proteins (Fig. 1c) and ribosome and translation regulation GTPases (Fig. 1d).

Next, we performed a separate screen to characterize the binding of the $B$. anthracis proteome to pppGpp (Fig. 1a). pppGpp is the predominant alarmone induced upon amino acid starvation in Bacillus species, rising to a higher level than ppGpp. However, despite potential differences in specificity between pppGpp and ppGpp, the pppGpp interactome has not been systematically characterized in bacteria. We used both His-tagged and HisMBP-tagged libraries to test pppGpp binding. Using two differentially tagged libraries allows us to identify more potential hits and minimize false negatives. We found that pppGpp shares almost identical targets with ppGpp, with similar or reduced binding efficacy for most of its targets compared to ppGpp (Supplementary Data 1). By sharing targets with ppGpp, pppGpp also comprehensively regulates purine synthesis and ribosome assembly. We also found that several proteins bind to pppGpp but not ppGpp, including the small alarmone synthetase YjbM (SAS1). This is expected for YjbM, since it is allosterically activated by pppGpp, but not $\mathrm{ppGpp}^{25}$.

NahA, a NuDiX hydrolase among the (p)ppGpp interactome in Bacillus, hydrolyzes (p)ppGpp to produce $\mathrm{pGpp}$ in vitro. The putative NuDiX hydrolase, BA5385, was identified as a novel binding target of (p)ppGpp. Protein sequence alignment showed that BA5385 has homologs in different Bacillus species with extensive homology and a highly conserved NuDiX box (Fig. S1). We cloned its homolog, YvcI, from the related species Bacillus subtilis and showed that overexpressed B. subtilis YvcI in cell lysate also binds ppGpp and pppGpp (Fig. 2a). The binding is highly specific, as non-radiolabeled ppGpp effectively competes with radiolabeled (p)ppGpp binding, whereas non-radiolabeled GTP failed to compete. EDTA eradicated (p)ppGpp binding to His-MBP-YvcI cell lysate, which implies that the divalent cation present in the reaction $\left(\mathrm{Mg}^{2+}\right)$ is essential for (p)ppGpp binding to YvcI (Fig. 2a).

We noticed that while YvcI-overexpression cell lysate showed strong and specific binding to (p)ppGpp, the purified protein does not appear to bind (p)ppGpp in DRaCALA (Fig. S2). This suggests that either YvcI requires a co-factor present in the lysate to bind to (p)ppGpp, or YvcI may rapidly hydrolyze (p) ppGpp and release the product. Therefore, we incubated purified YvcI with $\left[5^{\prime}-\alpha^{-32} \mathrm{P}\right]-(\mathrm{p}) \mathrm{ppGpp}$ and ran the reaction product using TLC (Fig. S3). We found that YvcI can hydrolyze both ppGpp and pppGpp. We also tested the ability of YvcI to hydrolyze GTP and 8-oxo-GTP to sanitize guanosine nucleotide $\mathrm{pool}^{26}$. YvcI failed to hydrolyze either GTP (Fig. S3a) or 8oxo-GTP (Fig. S3c). The inability of YvcI to hydrolyze GTP despite the structural similarity between GTP and (p)ppGpp suggest that NahA is a specific (p)ppGpp hydrolase which requires its substrate to have pyrophosphate group on the $3^{\prime}$ end. 
a

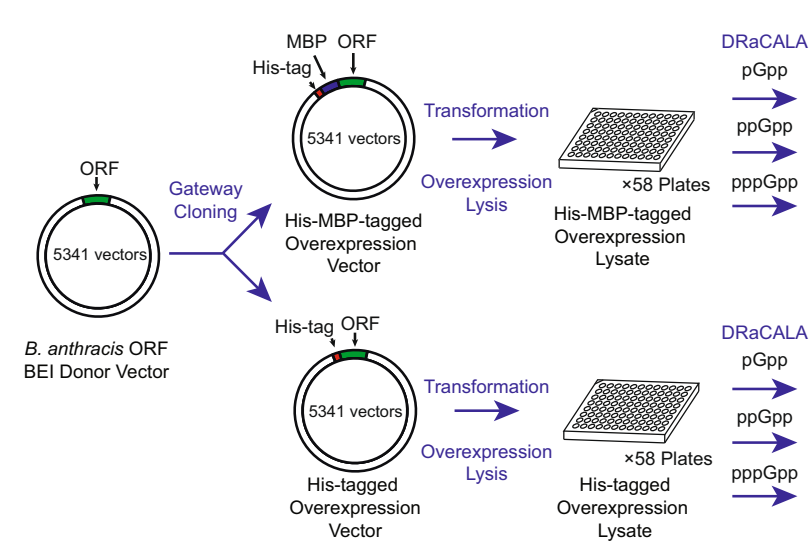

C

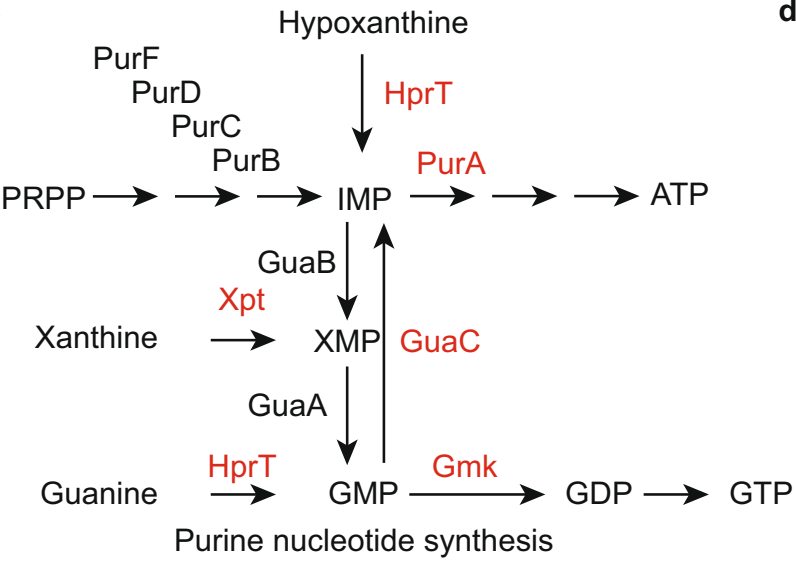

b

\begin{tabular}{|c|c|c|c|c|c|c|}
\hline \multirow{2}{*}{$\begin{array}{l}\text { B. anthracis } \\
\text { Locus Tag }\end{array}$} & \multirow{2}{*}{ Name } & \multicolumn{3}{|c|}{ B. anthracis (This Work) } & \multirow{2}{*}{$\begin{array}{l}\text { E. coli } 15,20 \\
\text { (p)ppGpp }\end{array}$} & \multirow{2}{*}{$\begin{array}{c}\text { S. aureus }{ }^{18} \\
\text { ppGpp }\end{array}$} \\
\hline & & pppGpp & ppGpp & pGpp & & \\
\hline \multirow{3}{*}{\multicolumn{7}{|c|}{$\begin{array}{l}\text { Purine } \\
\text { Synthesis } \\
\text { Pathway }\end{array}$}} \\
\hline & & & & & & \\
\hline & & & & & & \\
\hline BA0063 & HprT & + & + & + & $+^{15,20}$ & + \\
\hline BA1591 & Xpt & + & + & + & NA & \\
\hline BA4009 & Gmk & + & + & + & & + \\
\hline BA5716 & PurA & + & + & + & $+^{15}$ & \\
\hline BA5705 & GuaC & NT & + & + & & \\
\hline \multirow[t]{6}{*}{ BA0044 } & PurR & + & + & + & & \\
\hline & PurB & & & & $+^{15}$ & \\
\hline & Purc & & & & $+^{15}$ & \\
\hline & PurD & & & & $+^{15}$ & \\
\hline & PurF & & & & $+^{15}$ & \\
\hline & Gpt & NA & NA & NA & $++^{15,20}$ & \\
\hline
\end{tabular}

Ribosome and

Translation

Regulation

BA1828 HflX

BA1525
BA4672 Der

$\begin{array}{lll}\text { BA3976 } & \text { Rb } \\ \text { BA5734 } & \text { TrmE }\end{array}$

$\begin{array}{ll}\text { BA5734 } & \text { Trm } \\ \text { BA4524 } & \text { Era }\end{array}$

(p)ppGpp

Metabolism

BA4637 Rel(RelA)

BA1212

BA5043, BA5385 Nudix hydrolase

BA3162, BA4322 5'-nucleotidase

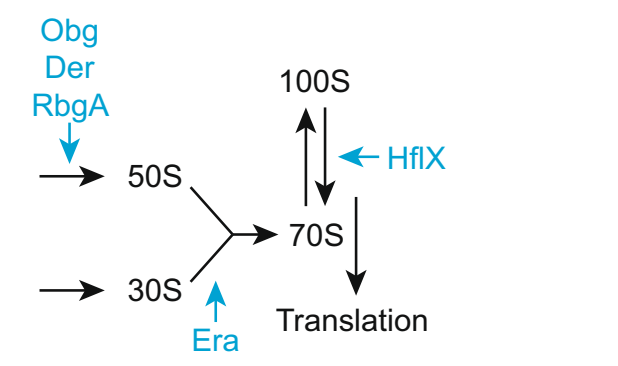

Ribosome biogenesis and translation regulation

Red: bound by pGpp, ppGpp and pppGpp

Blue: bound by ppGpp and pppGpp, but not by pGpp

Fig. 1 Proteome-wide DRaCALA screen identifies both conserved categories of binding targets and novel targets. a The Bacillus anthracis ORF donor vector library was recombined by Gateway cloning into overexpression vectors to generate ORFs with an N-terminal His-tag or HisMBP tag. The plasmids were transformed into $E$. coli for overexpression of recombinant proteins. Lysates of each ORF overexpressed in $E$. coli were assayed for binding to pppGpp, ppGpp, and pGpp using DRaCALA. b List of identified (p)ppGpp-binding targets in E. coli, S. aureus, and B. anthracis and pGpp-binding targets in B. anthracis. pppGpp results were obtained using both His-tagged and His-MBP-tagged proteins. ppGpp and pGpp results were obtained using His-MBP-tagged proteins. NT not tested, NA not available due to the lack of homologous gene. c, d Schematics of pathways differentially regulated by $p G p p$ and (p)ppGpp: c Enzymes in purine nucleotide synthesis, including HprT, Xpt, Gmk, and GuaC, bound both pGpp and (p)ppGpp; d GTPases, involved in ribosome biogenesis and translational control (Obg, $\mathrm{HflX}$, Der, RbgA, and Era), bound (p)ppGpp, but not pGpp.

pppGpp and ppGpp can be hydrolyzed by the Rel enzyme, traditionally referred as RelA in $B$. subtilis ${ }^{27-29}$, to produce GTP and GDP respectively. However, unlike Rel, YvcI hydrolyzed pppGpp and ppGpp to yield a single-nucleotide species that migrated differently than GTP (Fig. S3a) or GDP (Fig. S3b). To determine the identity of Yvcl's (p)ppGpp hydrolysis product, we analyzed the sample by liquid chromatography coupled with mass spectrometry (LC-MS), and compared to a pGpp standard produced by E. faecalis SAS (RelQ) in vitro ${ }^{22}$. The LC-MS profile revealed a peak of the same mass over charge ratio $(\mathrm{m} / \mathrm{z})$ as GTP but with a different retention time $(11.75 \mathrm{~min}$ versus $11.15 \mathrm{~min}$ for GTP). The retention time is the same as the pGpp standard (Fig. 2b), suggesting that the hydrolysis product of YvcI is pGpp.

The production of pGpp from (p)ppGpp agrees with the $\mathrm{NuDiX}$ hydrolase function, inferring that pppGpp and ppGpp are hydrolyzed between the $5^{\prime}-\alpha$ and $5^{\prime}-\beta$ phosphate groups to produce guanosine- $5^{\prime}$-monophosphate- ${ }^{\prime}$-diphosphate (pGpp). Therefore, we renamed the enzyme NuDiX alarmone hydrolase A (NahA).

It is possible, although unlikely, that NahA hydrolyzes the $3^{\prime}-\beta$ phosphate and the $5^{\prime}-\gamma$-phosphate to produce ppGp, which would run at the same retention time as pGpp in LC-MS. To distinguish these two possibilities, we analyzed the NahA cleavage products of $\left[3^{\prime}-\beta-{ }^{32} \mathrm{P}\right]$-pppGpp. If NahA cleaves between $5^{\prime}-\alpha$ and $\beta$ phosphates, the reaction would yield $\left[3^{\prime}-\beta-32 \mathrm{P}\right]-\mathrm{pGpp}$. In contrast, if NahA cleaves between $3^{\prime}-\alpha$ and $\beta$-phosphates, the reaction would yield free ${ }^{32} \mathrm{P}$-phosphate. TLC analysis revealed that the radioactive ${ }^{32} \mathrm{P}$ after NahA hydrolysis of $\left[3^{\prime}-\beta-32 \mathrm{P}\right]-$ pppGpp co-migrates with the pGpp nucleotide rather than the free phosphate that migrates to the very end of TLC plate (Fig. 2c). This result showed that the product has an intact $3^{\prime}$ pyrophosphate group, confirming the product to be pGpp rather 
a

$\begin{array}{rcccc}{ }^{32} \text { P-ppGpp } & + & + & + & + \\ \text { GTP } & - & + & - & - \\ \text { EDTA } & - & - & + & - \\ \text { ppGpp } & - & - & - & + \\ \text { Yvcl/NahA } & - & & & \end{array}$

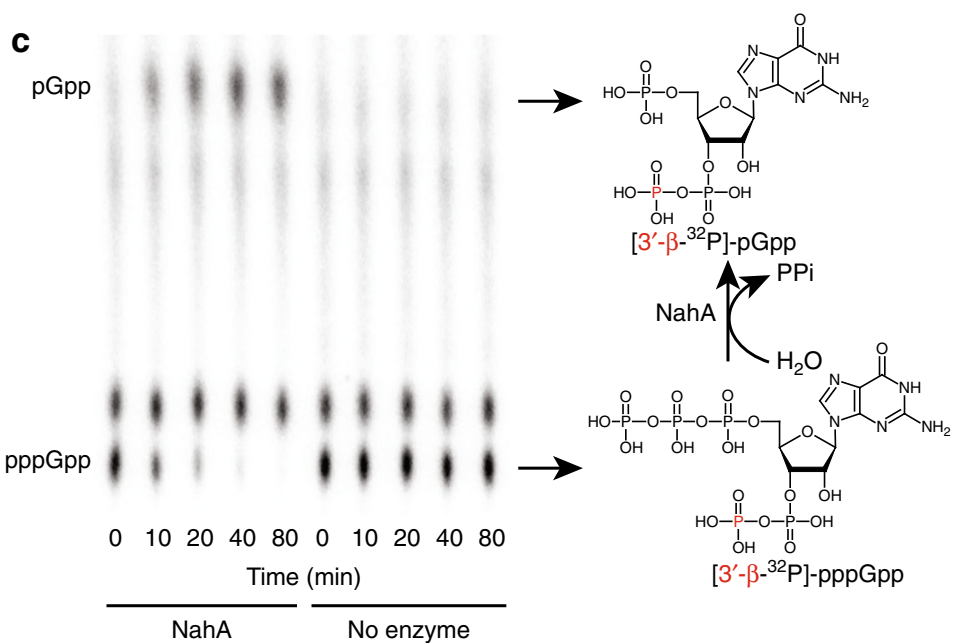

d

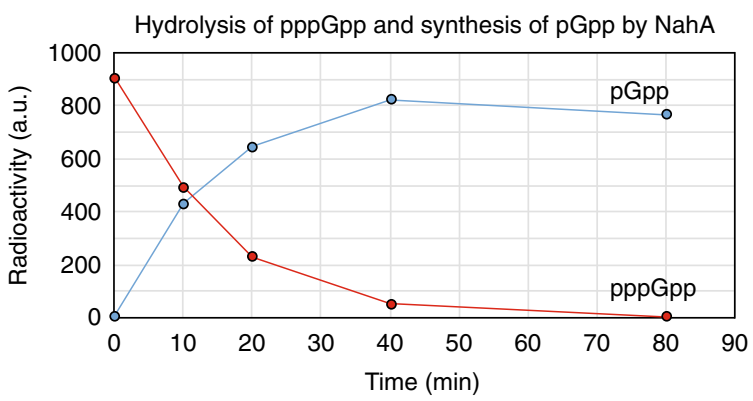

b

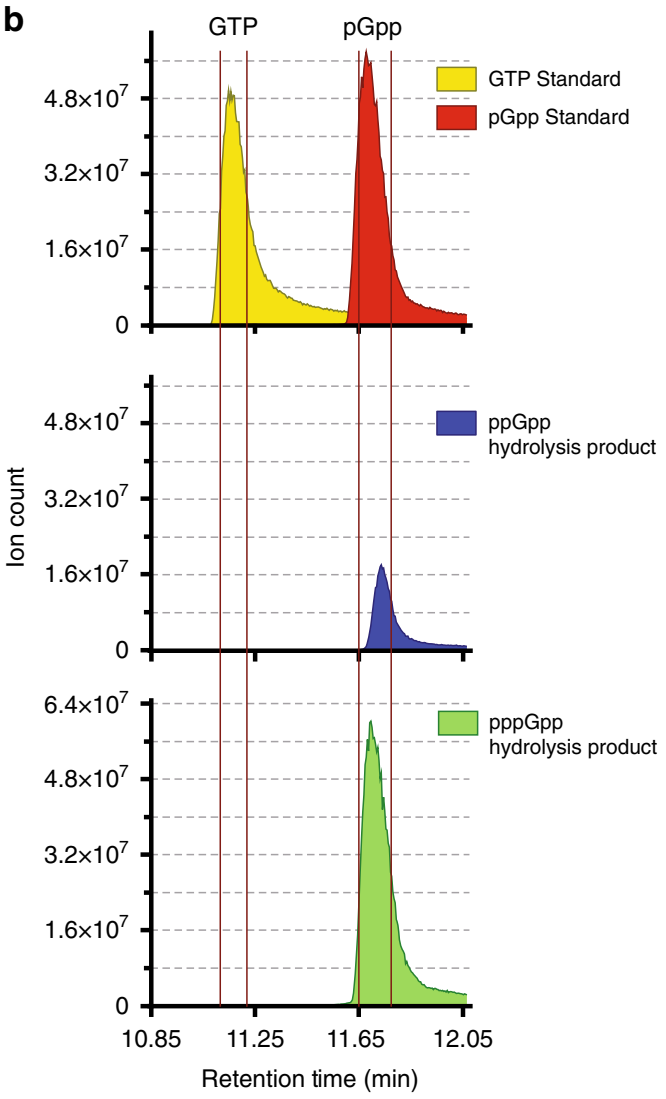

e

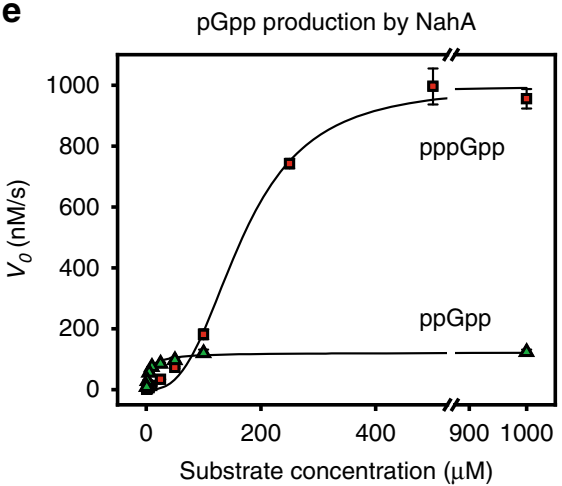

Fig. 2 NahA (Yvcl) produces pGpp via (p)ppGpp hydrolysis. a DRaCALA of [5'- $\alpha-32 \mathrm{P}]-\mathrm{ppGpp}$ binding to B. subtilis HisMBP-tagged Yvcl (NahA) overexpressed in E. coli cell lysate. Unlabeled GTP $(100 \mu \mathrm{M})$, unlabeled ppGpp $(100 \mu \mathrm{M})$ and EDTA $(10 \mathrm{mM})$ were added as indicated. $\mathbf{b}$ lon count vs. retention time curves from LC-MS of GTP and pGpp standards and NahA-catalyzed hydrolysis products from ppGpp and pppGpp. c TLC analysis of NahA activity over time with [3'- $\beta-32 \mathrm{P}]$-pppGpp. Expected NahA-catalyzed conversion of $\left[3^{\prime}-\beta-32 \mathrm{P}\right]-\mathrm{pppGpp}$ to $\left[3^{\prime}-\beta-32 \mathrm{P}\right]-\mathrm{pG} p$ is shown on the right. Radiolabeled $\left[3^{\prime}-\beta-32 \mathrm{P}\right]$-phosphorus atom is highlighted in red. $\mathbf{d}$ Quantitation of pppGpp and pGpp in the hydrolysis of pppGpp in c. e Initial velocity vs. pppGpp or ppGpp concentration curves for NahA synthesis of $\mathrm{pGpp}$. Data were obtained by kinetic assay using radiolabels (see Methods section). Curves represent the best fit of the data from three independent experiments. Error bars represent standard error of the mean.

than ppGp. Finally, quantification of $\left[3^{\prime}-\beta-32 \mathrm{P}\right]$-pppGpp hydrolysis by NahA showed that the decrease of substrate radioactivity mirrored the increase of the single product radioactivity (Fig. 2d), demonstrating the product is exclusively pGpp.

As a NuDiX hydrolase, NahA has been reported to have a modest activity in removing the $5^{\prime}$-phosphate of $\mathrm{mRNA}^{30}$. We found that NahA is far more efficient at hydrolyzing (p)ppGpp than at decapping mRNA. Enzymatic assays revealed that NahA hydrolyzes ppGpp following Michaelis-Menten kinetics, with a $k_{\text {cat }}$ of $1.22 \pm 0.17 \mathrm{~s}^{-1}$ and a $K_{\mathrm{m}}$ of $7.5 \pm 2.3 \mu \mathrm{M}$ (Fig. $2 \mathrm{e}$ and
Table 1 Kinetic parameters of (p)ppGpp hydrolysis by NahA.

\begin{tabular}{llc} 
& ppGpp & pppGpp \\
\hline$k_{\text {cat }}\left(\mathrm{s}^{-1}\right)$ & $1.22 \pm 0.17$ & $10.0 \pm 0.5$ \\
$K_{m}(\mu \mathrm{M})$ & $7.5 \pm 2.3$ & $177.4 \pm 0.4$ \\
Hill coefficient $n$ & 1 & $2.78 \pm 0.47$ \\
\hline
\end{tabular}


a

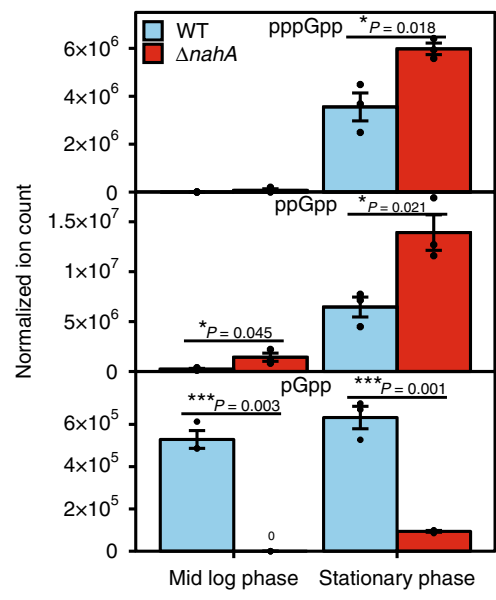

c

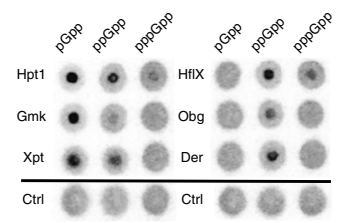

d
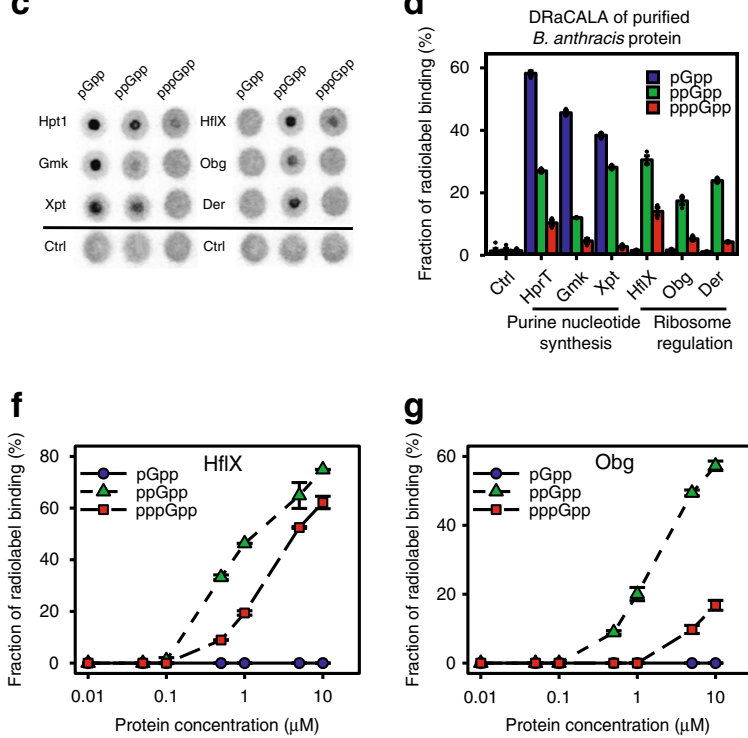

g

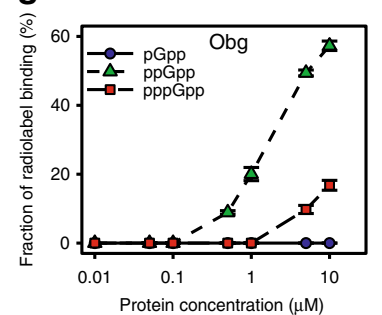

e

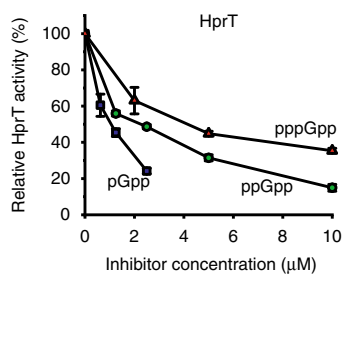

h

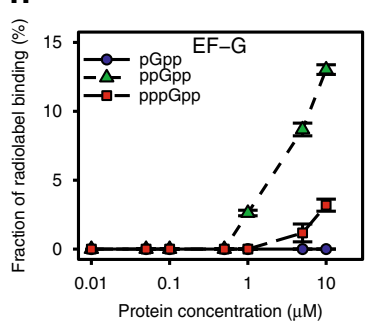

b

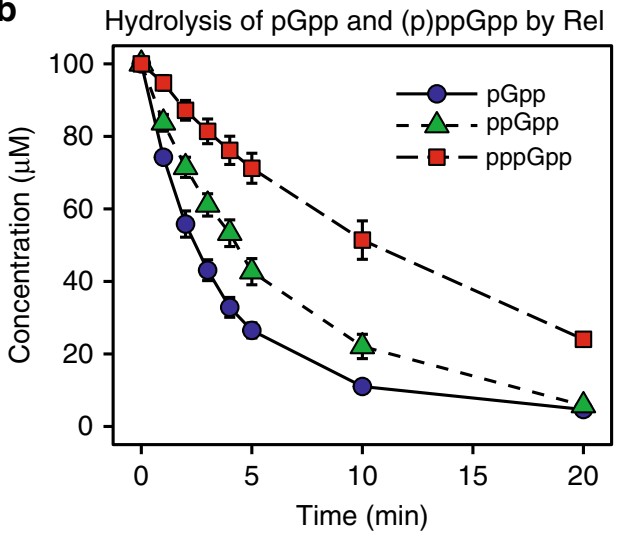

i

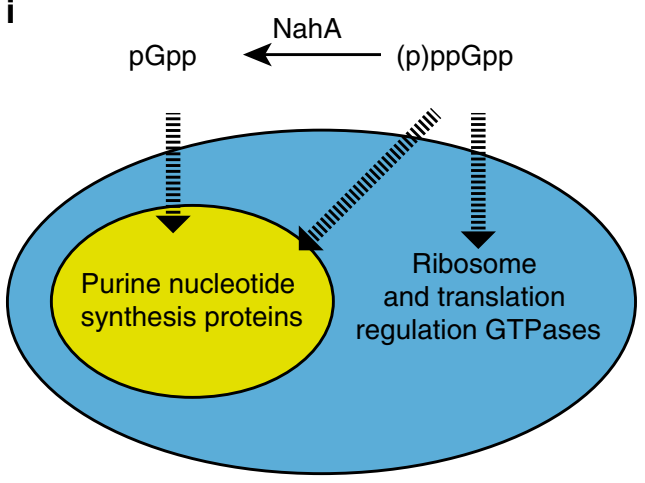

Fig. 3 pGpp is produced by NahA in vivo and has a distinct binding spectrum compared to (p)ppGpp. a LC-MS analyses of pGpp, ppGpp, and pppGpp of wild type and $\triangle n a h A$ in log phase and stationary phase. Normalized ion count is ion count per $\mathrm{OD}_{600 \mathrm{~nm}}$ per unit volume of the culture. Error bars represent standard errors of the mean of three biological replicates. A two-tailed two-sample equal-variance Student's $t$ test was performed between samples indicated by asterisks. Asterisks indicate statistical significance of differences $\left({ }^{\star} P \leq 0.05,{ }^{\star \star} P \leq 0.01,{ }^{\star \star \star} P \leq 0.001\right)$. b In vitro hydrolysis of pGpp, ppGpp, and pppGpp by B. subtilis Rel. For each nucleotide, $200 \mathrm{nM}$ Rel was incubated with a mix of $100 \mu \mathrm{M}$ unlabeled alarmone and a small amount of $5^{\prime}-\alpha^{-}{ }^{32} \mathrm{P}-$ labeled version. The degradation of the alarmone was analyzed by TLC to measure the decreased radioactivity. Error bars represent standard errors of the mean of three replicates. c DRaCALA of purified His-MBP-tagged B. anthracis proteins $(1 \mu \mathrm{M})$ with $<0.1 \mathrm{nM}$ of identical amount of $5^{\prime}-\alpha-32 \mathrm{p}-$ labeled $\mathrm{pG}$ - $\mathrm{pp}$, ppGpp, and pppGpp. d Quantification of DRaCALA in c. Error bars represent standard errors of the mean of three replicates except for control group (six replicates). e HprT enzymatic activities in the presence of indicated concentrations of pGpp, ppGpp, and pppGpp. The reaction was performed with 20 nM HPRT, $1 \mathrm{mM}$ PRPP, and $50 \mu \mathrm{M}$ guanine ${ }^{14}$. Error bars represent standard errors of the mean from three replicates. $\mathbf{f}-\mathbf{h}$ Titrations of $B$. anthracis GTPases and quantification of their binding to pGpp, ppGpp and pppGpp using DRaCALA: GTPases HfIX (f), Obg (g), and translation elongation factor $\mathrm{G}$ (EF-G) (h). Error bars represent standard errors of the mean of three replicates. i Schematic showing the relationship between pGpp-binding targets and ( $\mathrm{p}$ )ppGpp-binding targets.

Table 1). NahA also effectively and cooperatively hydrolyzes pppGpp, with a Hill coefficient of $2.78 \pm 0.47, k_{\text {cat }}$ of $10.0 \pm 0.5 \mathrm{~s}^{-1}$ and $K_{\mathrm{m}}$ of $177.4 \pm 0.4 \mu \mathrm{M}$ (Fig. $2 \mathrm{e}$ and Table 1). In contrast, its $k_{\text {cat }}$ to decap RNA is $\sim 0.0003 \mathrm{~s}^{-1}$ (estimation based on published figure) ${ }^{30}$. The difference in $k_{\text {cat }}$ in vitro suggests that NahA's major function is to regulate (p)ppGpp rather than to decap the $5^{\prime}$ cap of mRNA.

NahA hydrolyzes (p)ppGpp to produce pGpp in vivo. NahA was previously identified as a constitutively expressed protein with $\sim 600$ copies per cell ${ }^{31}$. To examine its impact on (p)ppGpp in vivo, we engineered a nahA deletion strain, and developed an LC-MS quantification for pppGpp, ppGpp, and pGpp in $B$. subtilis cells (see Methods section). LC-MS measurement of cell extracts showed that $\Delta n a h A$ cells accumulate more pppGpp and
ppGpp than wild-type cells during both log phase and stationary phase, in agreement with NahA's ability to hydrolyze (p)ppGpp (Fig. 3a). In contrast, $\triangle$ nahA mutant has much less pGpp than wild-type cells (Fig. 3a). Specifically, during log phase, pGpp can hardly be detected in $\triangle$ nahA (Fig. S4a). When we complement $\triangle n a h A$ with an overexpressed copy of nahA, the pGpp levels increased to more than wild-type levels (Figure S4). These results support the function of NahA in producing pGpp.

We also used the drug arginine hydroxamate (RHX) which mimics amino acid starvation to induce accumulation of $(p)$ $\mathrm{ppGpp}^{12}$. Using both LC-MS and TLC, we observed rapid accumulation of (p)ppGpp after RHX treatment, with $\Delta$ nahA cells showing stronger (p)ppGpp accumulation than wild-type cells (Fig. S5a-d). We still observed the accumulation of pGpp in $\Delta n a h A$ cells, although to a much less extent than wild-type cells 
(Fig. S5e). This remaining pGpp in $\triangle n a h A$ cells is likely due to the function of the enzyme SAS1, which can synthesize pGpp from GMP and $\mathrm{ATP}^{22}$.

pGpp can be hydrolyzed efficiently by B. subtilis RelA (Rel) in vitro. The strong reducing effect of NahA on (p)ppGpp levels suggests that (p)ppGpp are efficiently converted to pGpp. However, pGpp must be eventually degraded. In B. subtilis, pppGpp and ppGpp are hydrolyzed to GTP/GDP by RelA (Rel) enzyme, which has a functional (p)ppGpp hydrolase domain. Therefore, we performed in vitro assays using purified B. subtilis Rel and pGpp, ppGpp, or pppGpp. We found that all three nucleotides can be hydrolyzed efficiently by Rel, and pGpp was hydrolyzed more rapidly than (p)ppGpp (Fig. 3b).

Protein binding spectrum of pGpp is distinct from (p)ppGpp. To understand whether pGpp is just an intermediate of (p)ppGpp hydrolysis or is a bona fide alarmone with its own regulatory targets, we used DRaCALA to systematically screen the $B$. anthracis library for pGpp-binding targets (Fig. 1a, b and Supplementary Data 1). Our screen showed that pGpp binds strongly to multiple purine nucleotide synthesis enzymes (Fig. 1c), but to none of the (p)ppGpp-binding ribosome and translation regulation GTPases (Fig. 1d). We then purified selected pGpp and (p)
ppGpp-binding targets and tested with $\left[5^{\prime}-\alpha^{3}{ }^{32} \mathrm{P}\right]$-labeled pGpp, ppGpp, and pppGpp using DRaCALA (Fig. 3c, d). These results confirmed strong pGpp binding to guanosine nucleotide synthesis proteins (Hpt1, Gmk, and $\mathrm{Xpt}$ ). Enzyme kinetic assay of $\mathrm{Xpt}^{32}$ and HprT (Fig. 3e) confirmed that pGpp inhibited their activities more potently than ppGpp and pppGpp. In contrast, GTPases involved in ribosome biogenesis (HflX, Obg, Der) bind ppGpp but not pGpp (Fig. 3d). Titration analysis of these GTPases showed their strong affinity to ppGpp, modest affinity to pppGpp and lack of affinity to pGpp (Fig. $3 \mathrm{f}-\mathrm{h}$ and Supplementary Table 1). We conclude that among the two main groups of conserved interaction targets of (p)ppGpp, pGpp exclusively regulates the purine pathway, but not the GTPases, thus can serve as a specialized signal (Fig. 3i).

nahA mutant exhibits stronger inhibition of translation upon amino acid starvation, delayed outgrowth, and loss of competitive fitness. The fact that $\mathrm{pGpp}$ does not directly regulate ribosome biogenesis and translation implicates an in vivo function of NahA: reducing (p)ppGpp levels to alleviate translation inhibition upon stress, while still keeping purine biosynthesis in check. To test this hypothesis, we analyzed the effects of nahA on cellular growth and metabolism. We first compared the key metabolites (NTPs, NDPs, NMPs, nucleosides, and nucleobases)

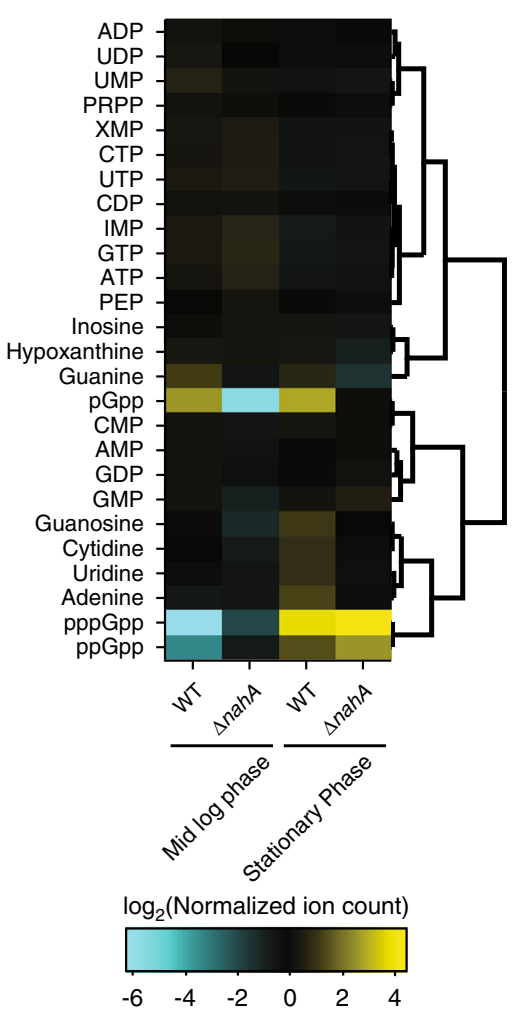

b

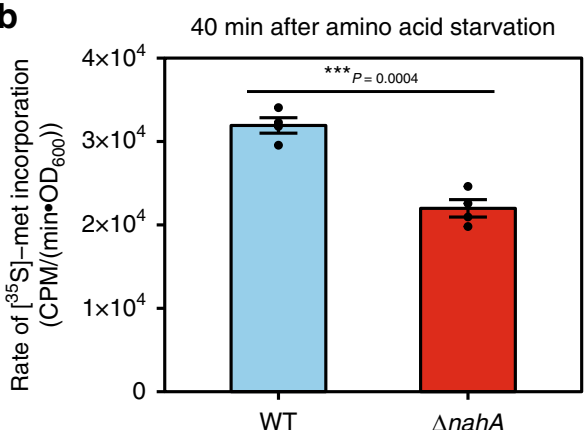

C

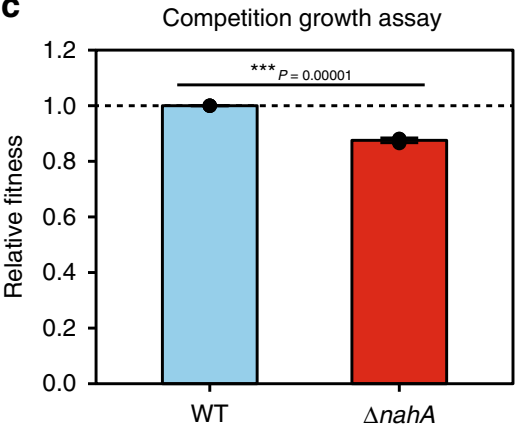

d

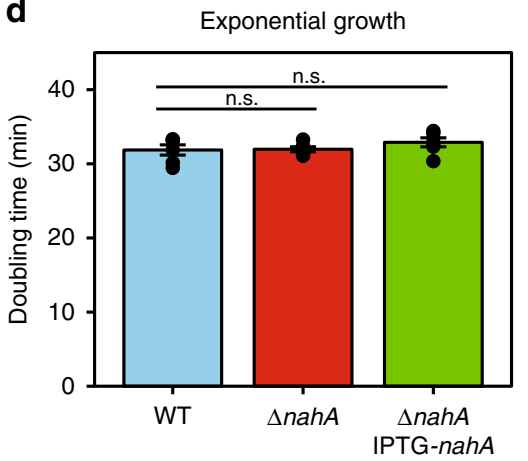

e

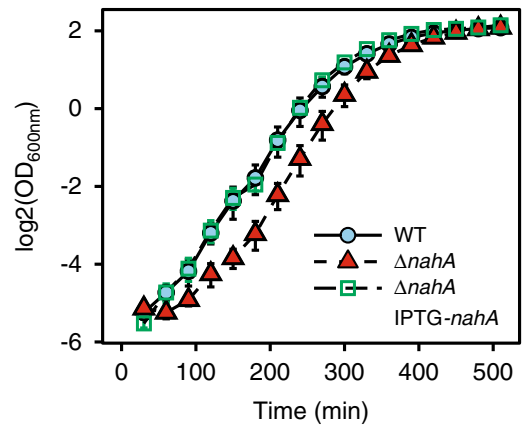

Fig. 4 The effect of NahA on purine nucleotides, translation, fitness, and growth recovery of $\boldsymbol{B}$. subtilis cells. a Hierarchical clustering of selected metabolites in exponential growth and stationary phase wild type and $\triangle$ nahA cells. Metabolites were measured by LC-MS. Normalized ion count is ion count per $\mathrm{OD}_{600 \mathrm{~nm}}$ per unit volume of the culture. $\mathbf{b}$ Protein translation rate of wild type and $\Delta$ nahA cells 40 min after amino acid starvation. Translation rate was measured by a 5 -min pulse of $\left[{ }^{35} \mathrm{~S}\right]$-methionine incorporation into TCA-precipitable fraction. Error bars represent standard errors of the mean from three replicates. $\mathbf{c}$ Relative fitness of $\triangle$ nahA and wild type strains obtained from a 7-day serial dilution competition assay. Error bar represents standard deviation from three repeats. $\mathbf{d}$ Doubling times of $\Delta n a h A$ and wild-type cells in log phase. A two-tailed two-sample equal-variance Student's $t$ test was performed between samples indicated by asterisks. Asterisk indicates statistical significance of difference $\left({ }^{\star} P \leq 0.05,{ }^{\star \star \star} P \leq 0.001, n . s\right.$. $\left.P>0.05\right)$. e Growth recovery from transient nutrient downshift. Log phase cultures of wild type, $\Delta$ nahA and nahA complementation ( $\triangle$ nahA IPTG-nahA) strains in rich media with 20 amino acids were firstly downshifted in minimum media without amino acids for 10 min, and then diluted in rich media for outgrowth. Result of growth recovery from similar downshift with the presence of guanosine is shown in Figure S6c. Error bars in d, e represent standard errors from six replicates. 
between wild-type cells and the nahA mutant, using LC-MS analyses of exponential growth and stationary phase cells (Fig. 4a and Supplementary Data 2). Despite much higher (p)ppGpp levels and much lower pGpp levels in the nahA mutant, most purine nucleotides exhibit very little difference, corroborating with the observation that $\mathrm{pGpp}$ regulates purine metabolism similarly to (p)ppGpp.

Next, we examined the effect of nahA on protein translation. We measured total protein translation rate of wild type and $\Delta$ nahA B. subtilis by pulsed incorporation of ${ }^{35}$ S-methionine (Fig. 4b). We induced amino acid starvation either by resuspending cells from amino acid replete medium to minimal medium, or by adding the nonfunctional amino acid analog arginine hydroxamate. In both cases, the rate of ${ }^{35} \mathrm{~S}$-methionine incorporation is significantly lower in $\Delta n a h A$ than in wild type, indicating a stronger inhibition of translation (Figs. $4 \mathrm{~b}$ and S6a).

Finally, we examined the impact of nahA on fitness of $B$. subtilis. We performed a growth competition assay in which a mixture of $\triangle$ nahA and wild-type cells were grown to saturation and then diluted repeatedly for days. The proportion of $\Delta n a h A$ rapidly decreased (Fig. S6b). The relative fitness of $\triangle n a h A$ is $0.876 \pm 0.009$ (mean \pm S.D.), indicating significant loss of fitness compared to wild-type cells (Fig. 4c). We found that $\Delta n a h A$ has a similar doubling time as wild-type cells (Fig. $4 \mathrm{~d}$ ), but a longer lag phase in adjusting to starvation (Fig. 4e). Together, these results suggest that NahA tunes alarmone composition and alarmone levels to promote B. subtilis adjustment to nutrient fluctuation and optimizes growth and fitness.

\section{Discussion}

Alarmones are universal stress signaling nucleotides in bacteria, however, the repertoire of alarmones and the target spectrums for each alarmone are incompletely understood. In this work, we have comprehensively characterized the interactomes of the related alarmones pppGpp and ppGpp and established the in vivo presence of pGpp as a closely related alarmone in Gram-positive Bacillus species. We characterized the direct targets of (p)ppGpp by screening an open reading frame expression library from $B$. anthracis. From this screen, we identified an enzyme NahA that converts (p)ppGpp to $\mathrm{pGpp}$ as efficient means to produce $\mathrm{pGpp}$ and to reduce (p)ppGpp concentrations, thus regulating the composition of the alarmones. We demonstrated that $\mathrm{pGpp}$ binds a distinct subset of protein receptors of (p)ppGpp. We also identified a key role of NahA in nutrient adaptation, suggesting that regulating alarmone composition may serve as a separationof-function strategy for optimal adaptation.

Conservation of pppGpp and ppGpp regulation of purine biosynthesis and ribosome biogenesis pathways across different species of bacteria. (p)ppGpp regulates diverse cellular targets that differ between different bacteria. For example, (p)ppGpp directly binds to RNA polymerase in E. coli to control the transcription of ribosomal and tRNA operons yet the (p)ppGppbinding sites on RNA polymerase are not conserved beyond proteobacteria ${ }^{4-8}$. Instead, (p)ppGpp accumulation in firmicutes strongly down-regulates synthesis of GTP, the exclusive transcription initiating nucleotides of rRNA and tRNA operons in firmicutes, to achieve a similar transcription control with different direct targets ${ }^{12,33,34}$. Therefore, identifying whether certain aspects of (p)ppGpp regulation are conserved among bacterial species is important for understanding the principles of bacterial survival and adaptation.

Our DRaCALA screen with a $B$. anthracis library revealed many ppGpp and pppGpp binding targets in this pathogenic Gram-positive bacterium. Comparing ppGpp-binding targets in
B. anthracis to $S$. aureus ${ }^{18}$ and E. coli ${ }^{15,20}$ identified novel targets but more importantly, revealed a clear theme of conservation. Most notably, (p)ppGpp in all three species binds to multiple proteins in two key pathways: (1) purine nucleotide synthesis, (2) translation-related GTPases including ribosome biogenesis factors.

The regulation of purine nucleotide synthesis in firmicutes includes well characterized targets Gmk and HprT whose regulation by (p)ppGpp protects Bacillus subtilis against nutrient changes like amino acid starvation and purine fluctuation, preventing cells from accumulating toxic high levels of intracellular GTP $12,14,35$. These also include new-found likely targets such as enzymes GuaC and PurA and the de novo pathway transcription factor PurR. Intriguingly, in the evolutionarily distant E. coli, the ppGpp targets in the purine biosynthesis pathway are different. For example, Gmk is not regulated by (p) ppGpp in $E$. coli $i^{35}$. On the other hand, (p)ppGpp directly targets the E. coli de novo enzyme PurF ${ }^{15}$, a target that is not conserved in firmicutes. Therefore, despite differences in precise targets, $(p)$ ppGpp extensively regulates the purine biosynthesis pathway in evolutionarily diverse bacteria (Fig. 1c), highlighting this critical physiological role of (p)ppGpp.

We also found that (p)ppGpp interacts with essential GTPases that are implicated in ribosome biogenesis and translation in $B$. subtilis and B. anthracis (Fig. 1d). (p)ppGpp's targets in GTPases from E. coli to firmicutes are conserved ${ }^{16-19}$ : HflX, Obg, and Era, are also (p)ppGpp-binding proteins in $E$. coli and $S$. aureus $^{15,18,20}$; Der and TrmE were identified in E. coli although not in S. aureus as (p)ppGpp-binding proteins ${ }^{15,20}$; RbgA does not exist in E. coli and it is identified as a (p)ppGpp-binding protein in S. aureus ${ }^{18}$. HflX is a ribosome-splitting factor which may rescue stalled ribosomes under stressed conditions ${ }^{36}$, and mediates the dissociation of hibernating $100 \mathrm{~S}$ ribosome to resume normal translation ${ }^{37}$. $\operatorname{Obg}^{16}, \operatorname{Der}^{38}$, and $\mathrm{RbgA}^{39,40}$ participate in the maturation of the $50 \mathrm{~S}$ subunit of ribosome. Era functions in the assembly of the functional $70 \mathrm{~S}$ ribosome complex ${ }^{41}$. TrmE functions in the maturation of tRNA to facilitate translation ${ }^{42}$. (p) ppGpp can be produced by amino acid starvation-induced translational stress or by defects in tRNA maturation ${ }^{43}$. Thus the conservation of (p)ppGpp regulation of GTPase targets highlights the key function of (p)ppGpp in quality control of ribosome biogenesis and regulation of translation.

The third major alarmone pGpp in Gram-positive species and its specific regulatory effect. In addition to pppGpp and ppGpp, it was long suspected that in B. subtilis, there are additional alarmones such as pppApp, pGpp, and ppGp accumulating during the stringent response ${ }^{44,45}$. Here we detected pGpp in $B$. subtilis cells, characterized an enzyme for its production in vivo and in vitro, and identified its targets. In contrast to our DRaCALA screens for pppGpp and ppGpp which identify similar targets and functionalities between these two alarmones, our DRaCALA screen for pGpp displays a strong difference from (p)ppGpp with regarding to two conserved pathways. The affinity and inhibitory potency of $\mathrm{pGpp}$ for purine nucleotide synthesis enzymes are equivalent or higher than that of (p)ppGpp. In contrast, pGpp's affinity to GTPases involved in translational regulation is much lower, or completely absent, compared to $(\mathrm{p})$ ppGpp. The distinct profile of target receptors establishes $\mathrm{pGpp}$ as a different alarmone from (p)ppGpp, allowing fine tuning of bacterial stress response. We propose a model for the function of NahA in growth recovery and competitive fitness by its role in transforming the alarmones. In wild-type cells, (p)ppGpp produced in response to amino acid starvation will be hydrolyzed in part by NahA to pGpp. (p)ppGpp concentration in wild-type cells 
during amino acid starvation can reach $>1 \mathrm{mM}$. Given the copy number of NahA as $\sim 600$ copies per cell and its maximum velocities for (p)ppGpp hydrolysis, (p)ppGpp concentrations observed in wild-type cells is a balance of synthesis by enzymes Rel and SAS1/2, and hydrolysis by Rel and NahA. Thus, in $\Delta n a h A$ cells, (p)ppGpp accumulates higher than in wild-type cells during amino acid starvation. Stronger inhibition of translation due to overly high level of (p)ppGpp in $\Delta n a h A$ leads to slower growth recovery after nutrient stress and thus leads to fitness loss when co-cultured with wild-type strain. In addition, because pGpp appears to be hydrolyzed more efficiently by RelA than ppGpp and pppGpp (Fig. 3b), another role of NahA is perhaps to speed up (p)ppGpp removal to promote growth recovery.

In E. coli, (p)ppGpp-binding proteins also include NuDiX hydrolases NudG and Mut ${ }^{20}$. Like NahA, NudG and MutT also hydrolyze (p)ppGpp. Unlike NahA, NudG and MutT produce guanosine $5^{\prime}$-monophosphate $3^{\prime}$-monophosphate (pGp) rather than pGpp. Similarly, in the bacterium Thermus thermophilus, the NuDiX hydrolase Ndx8 is also found to hydrolyze (p)ppGpp to produce $\mathrm{pGp}^{46}$. Sequence alignment shows that their homology with NahA is mostly restricted in the NuDiX box that is shared by NuDiX hydrolase family (Fig. S1). Therefore, NudG, MutT, and $\mathrm{Ndx} 8$ are considered alternative (p)ppGpp hydrolysis pathways to remove (p)ppGpp and promote growth ${ }^{46}$, rather than an alarmone-producer.

In E. coli, the enzyme GppA converts pppGpp to ppGpp, which may also regulate the composition of its alarmones ${ }^{21}$. In the absence of GppA, the alarmone pppGpp accumulates to a higher level than $\mathrm{ppGpp}^{47}$. It is possible that a similar separation-offunction regulation exists in $E$. coli by tuning pppGpp vs ppGpp levels. This can be addressed by examining pppGpp interactome in E. coli, which may reveal a differential theme than ppGpp. Ultimately, discovery of more enzymes that interconvert signaling molecules may reveal a universal theme of optimization through fine-tuning in signal transduction.

\begin{abstract}
Methods
Bacillus anthracis ORFeome Library Construction. Bacillus anthracis Gateway ${ }^{\circledast}$ Clone Set containing plasmids bearing $B$. anthracis open reading frames was acquired from BEI Resources and used for Gateway cloning (Invitrogen protocol) into overexpression vectors pVL791 ${ }^{48}$ (10xHis tag ampicillin-resistant) and pVL847 $7^{48}$ (10xHis-MBP tag, gentamycin-resistant) and transformed into Escherichia coli BL21 lacI' to produce two open reading frame proteome over-expression libraries (ORFeome library). The resulting ORFeome library contains 5139 ORFs from the genome of B. anthracis str. Ames (91.2\% of 5632 ORFs in the genome). The corresponding proteins were expressed in E. coli and the cells were lysed to prepare the overexpression lysates for the downstream analysis: $E$. coli strains containing overexpression vectors were grown in LB-M9 media $\left(7 \mathrm{~g} / \mathrm{L} \mathrm{Na}_{2} \mathrm{HPO}_{4}\right.$, $2 \mathrm{~g} / \mathrm{L} \mathrm{KH}_{2} \mathrm{PO}_{4}, 0.5 \mathrm{~g} / \mathrm{L} \mathrm{NaCl}, 1 \mathrm{~g} / \mathrm{L} \mathrm{NH} \mathrm{Cl}_{4} \mathrm{Cl} 2 \mathrm{~g} / \mathrm{L}$ glucose, $1 \mathrm{~g} / \mathrm{L}$ sodium succinate dibasic hexahydrate; $10 \mathrm{~g} / \mathrm{L}$ tryptone, $5 \mathrm{~g} / \mathrm{L}$ yeast extract, and $3 \mathrm{mM} \mathrm{MgSO}_{4}$ ) at $30{ }^{\circ} \mathrm{C}$ and the overexpression was induced by $0.5 \mathrm{mM}$ IPTG. Cells were collected by centrifugation at $5000 \times \mathrm{g} 4{ }^{\circ} \mathrm{C}$ for $10 \mathrm{~min}$. Cells were resuspended in $10 \%$ original volume of resuspension/lysis buffer $(10 \mathrm{mM}$ Tris $\mathrm{pH} 7.5,100 \mathrm{mM} \mathrm{NaCl}, 5 \mathrm{mM}$ $\mathrm{MgCl}_{2}, 0.25 \mathrm{mg} / \mathrm{mL}$ lysozyme, $0.01 \mathrm{mg} / \mathrm{mL}$ DNase I, and $1 \mathrm{mM}$ PMSF). Cells were then lysed by three rounds of $-80^{\circ} \mathrm{C}$ freezing and room temperature thawing.
\end{abstract}

Plasmid and strain construction. Plasmid for NahA purification was constructed as follows. To generate a His-SUMO-NahA plasmid, the nahA sequence was PCR amplified using primers oJW3519/oJW3520 and incorporated into pE-SUMO vector backbone amplified using primers oJW3194/oJW3195 by Golden Gate assembly method (New England BioLabs) to generate pJW739.

To generate a His-MBP-NahA plasmid, the nahA sequence was PCR amplified using primers oJW3274/oJW3275 and incorporated into pVL847 ${ }^{48}$ (His-MBP-tag overexpression vector) by Gateway cloning method (Invitrogen) to generate pJW742.

$\Delta$ nahA::erm mutant of Bacillus subtilis was constructed by transformation of PCR product of BKE34780 (from BGSC) genome DNA amplified by oJW3382/ oJW3383 and erythromycin-lincomycin selection.

$\triangle$ nahA markerless deletion of Bacillus subtilis was constructed by CRISPR/Cas9 editing method ${ }^{49}$ : CRISPR editing plasmid pJW737 was constructed by Golden Gate assembly method (New England BioLabs) using pPB41 backbone, guide RNA template, and DNA fragments downstream and upstream of nahA. pPB41 ${ }^{49}$
Table 2 Strains used in this work.

ID Genotype

Ref.

DK1042 Bacillus subtilis NCIB 3610 comlQ12L (wild type)

JDW4085 Bacillus subtilis NCIB 3610 comlQ12L $\Delta$ nahA::erm

JDW4087 Bacillus subtilis NCIB $3610 \mathrm{comlQ12L} \Delta$ nahA

JDW4088 Bacillus subtilis NCIB $3610 \mathrm{coml}{ }^{\text {Q12L }} \Delta$ nahA amyE::Phyperspank::nahA

This work This work

This work

Table 3 Plasmids used in this work.

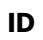
Construct

Source

pJW736

pJW737

pJW739

pJW742

pDR111 amyE::Phyperspank::nahA (amp, spec)

This work pPB41-gRNA(nahA)- $\Delta$ nahA (amp, spec) $\mathrm{pE}$-SUMO-Bacillus subtilis nahA (kan) pVL847-Bacillus subtilis nahA (gentamycin)

This work

This work

This work

backbone was amplified by oJW2775/oJW2821. Guide RNA template was made by annealing oJW3501/oJW3502. B. subtilis DNA fragments upstream and downstream of nahA were amplified by oJW3498/oJW3500 and oJW3497/ oJW3499, respectively. pJW737 was transformed into wild type B. subtilis followed $30^{\circ} \mathrm{C}$ spectinomycin selection. Transformants were sequentially patched and grown on LB agar without antibiotics at $45^{\circ} \mathrm{C}$ to remove the plasmid pJW737. Colonies cured of pJW737 (sensitive to spectinomycin) were PCR verified using oJW3382/oJW3383 and the colonies with PCR product of $1422 \mathrm{bp}$ were $\triangle n a h A$.

To generate an IPTG-inducible nahA in B. subtilis, the nahA sequence was PCR amplified using primers oJW3400/oJW3401 and incorporated into pDR111 ${ }^{50}$ by restriction cut by SalI and SphI followed by T4 DNA ligase mediated ligation (New England BioLabs) to generate pJW736. $\Delta$ nahA amyE::Phyperspank::nahA ( $\Delta$ nahA IPTG-nahA) mutant of Bacillus subtilis was constructed by transformation of pJW736 into $\triangle$ nahA mutant and spectinomycin selection.

Strains, plasmids, and primers are listed in (Tables 2-4).

Growth conditions. If not specifically mentioned, B. subtilis strains were grown in S7 defined media ${ }^{51}$ with modifications (50 mM MOPS instead of $100 \mathrm{mM}, 0.1 \%$ potassium glutamate, $1 \%$ glucose, no additional amino acids, and nucleosides or nucleobases), with shaking at $250 \mathrm{rpm} 37^{\circ} \mathrm{C}$. The modified S7 defined media with 20 amino acids contains $50 \mu \mathrm{g} / \mathrm{mL}$ alanine, $50 \mu \mathrm{g} / \mathrm{mL}$ arginine, $50 \mu \mathrm{g} / \mathrm{mL}$ asparagine, $50 \mu \mathrm{g} / \mathrm{mL}$ glutamine, $50 \mu \mathrm{g} / \mathrm{mL}$ histidine, $50 \mu \mathrm{g} / \mathrm{mL}$ lysine, $50 \mu \mathrm{g} / \mathrm{mL}$ proline, $50 \mu \mathrm{g} / \mathrm{mL}$ serine, $50 \mu \mathrm{g} / \mathrm{mL}$ threonine, $50 \mu \mathrm{g} / \mathrm{mL}$ glycine, $50 \mu \mathrm{g} / \mathrm{mL}$ isoleucine, 50 $\mu \mathrm{g} / \mathrm{mL}$ leucine, $50 \mu \mathrm{g} / \mathrm{mL}$ methionine, $50 \mu \mathrm{g} / \mathrm{mL}$ valine, $50 \mu \mathrm{g} / \mathrm{mL}$ phenylalanine, $500 \mu \mathrm{g} / \mathrm{mL}$ aspartic acid, $500 \mu \mathrm{g} / \mathrm{mL}$ glutamic acid, $20 \mu \mathrm{g} / \mathrm{mL}$ tryptophan, $20 \mu \mathrm{g} / \mathrm{mL}$ tyrosine, and $40 \mu \mathrm{g} / \mathrm{mL}$ cysteine.

Nucleotide preparation. pppGpp was synthesized in vitro from $8 \mathrm{mM}$ ATP and 6 $\mathrm{mM}$ GTP using RelSeq ${ }_{1-385^{21}}$. For production of $\left[5^{\prime}-\alpha^{-32} \mathrm{P}\right]-\mathrm{pppGpp}, 750 \mu \mathrm{Ci} / \mathrm{mL}$ ${ }^{32} \mathrm{P}-\alpha$-GTP $(3000 \mathrm{mCi} / \mathrm{mmol}$; PerkinElmer) was used instead of non-radiolabeled GTP. For production of $\left[3^{\prime}-\beta-{ }^{32} \mathrm{P}\right]-\mathrm{pppGpp}, 750 \mu \mathrm{Ci} / \mathrm{mL}^{32} \mathrm{P}-\gamma-\mathrm{ATP}(3000 \mathrm{mCi} /$ mmol; PerkinElmer) was used instead of non-radiolabeled ATP. ppGpp was synthesized from pppGpp using $\mathrm{GppA}^{21}$. For purification of nucleotides, the reaction mix was diluted in Buffer A (0.1 mM LiCl, $0.5 \mathrm{mM}$ EDTA, $25 \mathrm{mM}$ Tris $\mathrm{pH} 7.5)$. The mixture was loaded onto a Buffer-A-equilibrated $1 \mathrm{~mL}$ HiTrap QFF column (GE Healthcare). The column was washed by 10 column volumes of Buffer A, followed by 10 column volumes of Buffer A with $170 \mathrm{mM} \mathrm{LiCl}$ for pppGpp purification (for ppGpp purification, the $\mathrm{LiCl}$ concentration was $160 \mathrm{mM}$ ). Radiolabeled (p)ppGpp was eluted by 5 column volumes of Buffer A with $500 \mathrm{mM} \mathrm{LiCl}$ with $1 \mathrm{~mL}$ fractions.

pGpp was synthesized from pppGpp in vitro using NahA in a reaction containing $25 \mathrm{mM}$ bis-Tris propane $\mathrm{pH} 9.0,15 \mathrm{mM} \mathrm{MgCl}_{2}, 75 \mathrm{mM} \mathrm{NH}_{4} \mathrm{Cl}$ and 0.1 $\mathrm{mg} / \mathrm{mL}$ NahA. For purification, we diluted the reaction mix in Buffer $\mathrm{A}$. The mixture was loaded onto a Buffer-A-equilibrated $1 \mathrm{~mL}$ HiTrap QFF column (GE Healthcare), washed by 10 column volumes of Buffer $\mathrm{A}$, and eluted by 10 column volumes of Buffer A with $155 \mathrm{mM} \mathrm{LiCl}$ with $1 \mathrm{~mL}$ fractions, followed by 5 column volumes of Buffer A with $500 \mathrm{mM} \mathrm{LiCl}$ with $1 \mathrm{~mL}$ fractions. The radioactivity and purity of radiolabeled nucleotides were analyzed by thin-layer chromatography and phosphorimaging.

Overexpression and purification of NahA. His-tagged $\mathrm{NahA}$ was purified by $\mathrm{Ni}$ NTA affinity column, followed by SUMO protease cleavage to remove the tag. To express His-tagged protein, His-SUMO-NahA verctor (pJW739) was transformed into E. coli BL21(DE3) lacIq by chemical transformation. A single colony of the corresponding strain was grown in LB with $30 \mu \mathrm{g} / \mathrm{mL}$ kanamycin overnight. 
Table 4 Primers used in this work.

ID

oJW2775

oJW2821

oJW3194

oJW3195

oJW3274

oJW3275

oJW3382

oJW3383

oJW3400

oJW3401

oJW3497

oJW3498

oJW3499

oJW3500

oJW3501

oJW3502

oJW3519

oJW3520
Sequence

GACGGTCTCAGCTGGCTGTAGGCATAGGCTTGGTTATG

GTAGGTCTCTAAGGATTTCGCGGGATCGAGATCCTGCATTAATG

GCGGGTCTCAACCTCCAATCTGTTCGCGGTGAGCC

GCGGGTCTCATAATCGAGCACCACCACCACCACCA

GGGGACAAGTTTGTACAAAAAAGCAGGCTGGGTGACGTACTTGCAAAGAGTGACAAATTG

GGGGACCACTTTGTACAAGAAAGCTGGGTCTATTTGATGTGCTGCGGGTCTAAACGAT

GCTCAAAGTATTCTTCAAGCGAGAG

CATTCCACTTCATGACGTAAGAGG

CCCGTCGACAAAGGAGGTGTACATGTGACGTACTTGCAAAGAGTGACAAATTGT

CCCGCATGCCTATTTGATGTGCTGCGGGTCTAAACGATA

CAGGGTCTCACCTTCAAGCGGCAGGCCAGTCGCTGCCAGCGGAT

GTCGGTCTCACAGCCCAAATCGTAACGGCTACAGGAGACGGAAG

GTCGGTCTCTTTAGAAAGACAAGTCAGGGGGGAGAAAGA

GACGGTCTCACTAACCTTCGTCCTGTCATCGTCTCTTTAT

GACGGTCTCAAAACATACCAGTCTCTTCTCTGTACTCTCTGATGAGTTTTAGAGACCGTC

GACGGTCTCTAAAACTCATCAGAGAGTACAGAGAAGAGACTGGTATGTTTTGAGACCGTC

GTGGGTCTCTAGGTGTGACGTACTTGCAAAGAGTGACAAATTGT

GTGGGTCTCTATTATTTGATGTGCTGCGGGTCTAAACGATA
Overnight culture was 1:100 diluted in LB-M9 media with $30 \mu \mathrm{g} / \mathrm{mL}$ kanamycin and grown at $30^{\circ} \mathrm{C}$ with shaking for $4 \mathrm{~h}$. After adding $1 \mathrm{mM}$ IPTG for induction, the culture was further grown at $30^{\circ} \mathrm{C}$ for $4 \mathrm{~h}$. Then the culture was centrifuged at $4000 \times g$ for $30 \mathrm{~min}$ at $4^{\circ} \mathrm{C}$ and the supernatant was discarded. The pellet was stored at $-80^{\circ} \mathrm{C}$ before cell lysis. All the following steps were performed at $4{ }^{\circ} \mathrm{C}$. Pellet was resuspended in Lysis Buffer $(50 \mathrm{mM}$ Tris- $\mathrm{HCl} \mathrm{pH} \mathrm{8,10 \%} \mathrm{sucrose} \mathrm{w/v,} \mathrm{and} 300$ $\mathrm{mM} \mathrm{NaCl}$ ) and lysed by French press. Cell lysate was centrifuged at 15,000 rpm for $30 \mathrm{~min}$, and the supernatant was collected, filtered through $0.45-\mu \mathrm{m}$ pore-size cellulose syringe filter. His-SUMO-NahA filtered supernatant was purified using a 5-mL HisTrap FF column (GE Healthcare) equipped on an ÄKTA FPLC apparatus (GE Healthcare). SUMO Buffer A (50 mM Tris- $\mathrm{HCl}, 25 \mathrm{mM}$ imidazole, $500 \mathrm{mM}$ $\mathrm{NaCl}$, and $5 \%$ glycerol v/v) was used as washing buffer, and SUMO Buffer C (50 $\mathrm{mM}$ Tris- $\mathrm{HCl}, 500 \mathrm{mM}$ imidazole, $500 \mathrm{mM} \mathrm{NaCl}$, and $5 \%$ glycerol v/v) was used as the elution buffer. Fractions containing most abundant His-SUMO-NahA were combined with $100 \mu \mathrm{g}$ SUMO Protease and dialyzed twice against SUMO Protease Buffer $(50 \mathrm{mM}$ Tris- $\mathrm{HCl}, 500 \mathrm{mM} \mathrm{NaCl}, 5 \%$ glycerol v/v, and $1 \mathrm{mM} \beta$-mercaptoethanol) overnight. His-SUMO tag was removed by flowing through HisTrap FF column and collecting the flow-through. Purified NahA was analyzed by SDS-

PAGE and the concentration was measured by the Bradford assay (Bio-rad).

His-MBP-NahA was prepared using similar protocol as mentioned above with modifications: pJW742 was transformed into E. coli and all the media contain 10 $\mu \mathrm{g} / \mathrm{mL}$ gentamycin instead of kanamycin; SUMO-protease and His-SUMO tag removal were not applied.

Differential radial capillary action ligand assay. Cell lysate with overexpressed protein and purified protein were used for DRaCALA. In all, $10 \mu \mathrm{L}$ cell lysate or diluted, purified protein was mixed with $10 \mu \mathrm{L}$ diluted $\left[5^{\prime}-\alpha^{-32} \mathrm{P}\right]-(\mathrm{p}) \mathrm{ppGpp}$ or $\left[5^{\prime}-\right.$ $\left.\alpha^{32} \mathrm{P}\right]$-pGpp $(\sim 0.2 \mathrm{nM})$ in a buffer containing $10 \mathrm{mM}$ Tris $\mathrm{pH} 7.5,100 \mathrm{mM} \mathrm{NaCl}$ and $5 \mathrm{mM} \mathrm{MgCl}_{2}$, incubated at room temperature for $10 \mathrm{~min}$. In total, $\sim 2 \mu \mathrm{L}$ mixture was blotted onto nitrocellulose membrane (Amersham; GE Healthcare) and allowed for diffusion and drying. The nitrocellulose membrane loaded with mixture was exposed on phosphor screen, which was scanned by a Typhoon FLA9000 scanner (GE Healthcare). Fraction of (p)ppGpp binding $F$ was analyzed using the following equation ${ }^{24}$ :

$$
F=\frac{I_{\text {in }}-I_{\text {bkg }}}{I_{\text {tot }}}
$$

where $I_{\text {in }}$ is the intensity of the inner region of radioactivity pattern, $I_{\mathrm{bkg}}$ is the background intensity in the inner region representing the unbound ligands, and $I_{\text {tot }}$ is the total intensity of the whole radioactivity pattern. $I_{\mathrm{bkg}}$ can be calculated by the following equation:

$$
I_{\mathrm{bkg}}=A_{\mathrm{in}} \times \frac{I_{\mathrm{tot}}-I_{\mathrm{in}}}{A_{\mathrm{tot}}-A_{\mathrm{in}}}
$$

where $A_{\text {in }}$ is the area of the inner region of radioactivity pattern, and $A_{\text {tot }}$ is the total area of the whole radioactivity pattern.

Quantification of intracellular nucleotides by thin-layer chromatography. Cells were grown in low phosphate S7 defined media $(0.5 \mathrm{mM}$ phosphate instead of 5 $\mathrm{mM})$, labeled with $50 \mu \mathrm{Ci} / \mathrm{mL}$ culture ${ }^{32} \mathrm{P}$-orthophosphate at an optical density at $600 \mathrm{~nm}\left(\mathrm{OD}_{600 \mathrm{~nm}}\right)$ of $\sim 0.01$ and grown for two to three additional generations before sampling. Nucleotides were extracted by adding $100 \mu \mathrm{L}$ of sample into $20 \mu \mathrm{L}$ $2 \mathrm{~N}$ formic acid on ice for at least $20 \mathrm{~min}$. Extracted samples were centrifuged at $15,000 \times \mathrm{g}$ for $30 \mathrm{~min}$ to remove cell debris. Supernatant samples were spotted on
PEI cellulose plates (EMD-Millipore) and developed in $1.5 \mathrm{M}$ or $0.85 \mathrm{M}$ potassium phosphate monobasic $\left(\mathrm{KH}_{2} \mathrm{PO}_{4}\right)(\mathrm{pH}$ 3.4). TLC plates were exposed on phosphor screens, which were scanned by a Typhoon FLA9000 scanner (GE Healthcare). Nucleotide levels were quantified by ImageJ (NIH). Nucleotide levels are normalized to ATP level at time zero.

Kinetic assay of pppGpp, ppGpp, and pGpp hydrolysis. Hydrolysis reaction was performed at $37^{\circ} \mathrm{C}$. For NahA hydrolysis of (p)ppGpp, $100 \mathrm{nM}$ purified NahA was added to a reaction mix containing $40 \mathrm{mM}$ Tris- $\mathrm{HCl} \mathrm{pH} \mathrm{7.5,100} \mathrm{mM} \mathrm{NaCl,} 10 \mathrm{mM}$ $\mathrm{MgCl}_{2}$, indicated concentrations of non-radioactive and ${ }^{32} \mathrm{P}$-radiolabeled $(\mathrm{p})$ ppGpp. For (p)ppGpp and pGpp hydrolysis by B. subtilis RelA, $200 \mathrm{nM}$ purified RelA enzyme was added to a reaction mix containing $25 \mathrm{mM}$ Tris- $\mathrm{HCl} \mathrm{pH} 7.5,1$ $\mathrm{mM} \mathrm{MnCl}{ }_{2}, 100 \mu \mathrm{M}$ non-radioactive, and $\sim 0.2 \mathrm{nM}{ }^{32} \mathrm{P}$-radiolabeled pppGpp, ppGpp, or pGpp.

At each indicated time points after the reaction started, $10 \mu \mathrm{L}$ reaction mix was aliquoted into $10 \mu \mathrm{L}$ ice-chilled $0.8 \mathrm{M}$ formic acid. Samples at each time point were resolved by thin-layer chromatography on PEI-cellulose plates with $1.5 \mathrm{M} \mathrm{KH}_{2} \mathrm{PO}_{4}$ ( $\mathrm{pH}$ 3.4). Nucleotide levels were quantified as mentioned above and the phosphorimager counts of substrate and product were used to calculate the concentration of product by a formula:

$$
c_{\mathrm{P}}=c_{\mathrm{S} 0} \cdot \frac{V_{\mathrm{P}}}{V_{\mathrm{P}}+V_{\mathrm{S}}}
$$

Here $c_{\mathrm{p}}$ was the concentration of product, $c_{\mathrm{S} 0}$ was the concentration of substrate before the reaction starts, $V_{\mathrm{p}}$ was the phosphorimager count of product, and $V_{S}$ was the phosphorimager count of substrate. Initial rates of hydrolysis $\left(v_{0}\right)$ were calculated using the slope of the initial linear part of $c_{\mathrm{p}}$ over time curve at different initial substrate concentrations. Michaelis-Menten constant $\left(K_{\mathrm{m}}\right)$ and catalytic rate constant $\left(k_{\text {cat }}\right)$ were obtained by fitting the data of $v_{0}-c_{\mathrm{S} 0}$ to the model

$$
v_{0}=\frac{c_{\mathrm{E}} \cdot k_{\mathrm{cat}} \cdot c_{\mathrm{s} 0}^{n}}{K_{\mathrm{m}}^{n}+c_{\mathrm{s} 0}^{n}}
$$

by MATLAB (R2016b), where $c_{\mathrm{E}}$ was the concentration of NahA, $c_{\mathrm{S} 0}$ was the initial concentration of substrate, and $n$ was the Hill's coefficient (for ppGpp hydrolysis, fix $n$ to 1$)$.

LC-MS quantification of metabolites. Cells were grown to designated $\mathrm{OD}_{600 \mathrm{~nm}}$ In all, $5 \mathrm{~mL}$ of cultures were sampled and filtered through PTFE membrane (Sartorius) at time points before and after $0.5 \mathrm{mg} / \mathrm{mL}$ arginine hydroxamate treatment. Membranes with cell pellet were submerged in $3 \mathrm{~mL}$ extraction solvent mix (on ice 50:50 (v/v) chloroform/water) to quench metabolism, lyse the cells and extract metabolites. Mixture of cell extracts were centrifuged at $5000 \times g$ for $10 \mathrm{~min}$ to remove organic phase, then centrifuged at $20,000 \times g$ for $10 \mathrm{~min}$ to remove cell debris. Samples were frozen at $-80^{\circ} \mathrm{C}$ if not analyzed immediately. Samples were analyzed using an HPLC-tandem MS (HPLC-MS/MS) system consisting of a Vanquish UHPLC system linked to heated electrospray ionization (ESI, negative mode) to a hybrid quadrupole high resolution mass spectrometer (Q-Exactive orbitrap, Thermo Scientific) operated in full-scan selected ion monitoring (MSSIM) mode to detect targeted metabolites based on their accurate masses. MS parameters were set to a resolution of 70,000, an automatic gain control (AGC) of $1 \mathrm{e} 6$, a maximum injection time of $40 \mathrm{~ms}$, and a scan range of $90-1000 \mathrm{mz}$. LC was performed on an Aquity UPLC BEH C18 column $(1.7 \mu \mathrm{m}, 2.1 \times 100 \mathrm{~mm}$; Waters). Total run time was $30 \mathrm{~min}$ with a flow rate of $0.2 \mathrm{~mL} / \mathrm{min}$, using Solvent A $(97: 3$ (v/ v) water/methanol, $10 \mathrm{mM}$ tributylamine $\mathrm{pH} \sim 8.2-8.5$ adjusted with $\sim 9 \mathrm{mM}$ acetic 
acid) and $100 \%$ acetonitrile as Solvent $\mathrm{B}$. The gradient was as follows: 0 min, $5 \% \mathrm{~B}$; $2.5 \mathrm{~min}, 5 \% \mathrm{~B} ; 19 \mathrm{~min}, 100 \% \mathrm{~B} ; 23.5 \mathrm{~min} 100 \% \mathrm{~B} ; 24 \mathrm{~min}, 5 \% \mathrm{~B}$; and $30 \mathrm{~min}, 5 \% \mathrm{~B}$. Raw output data from the MS was converted to mzXML format using inhousedeveloped software, and quantification of metabolites were performed by using the Metabolomics Analysis and Visualization Engine (MAVEN 2011.6.17, http:// genomics-pubs.princeton.edu/mzroll/index.php) software suite ${ }^{52,53}$. Normalized ion count was defined and calculated as the ion count per $\mathrm{OD}_{600 \mathrm{~nm}}$ per unit volume $(5 \mathrm{~mL})$ of the culture.

Growth competition assay. nahA: :erm ${ }^{R}$ mutant and wild-type cells were mixed in $\mathrm{LB}$ broth to an $\mathrm{OD}_{600 \mathrm{~nm}}$ of 0.03 and grown at $37^{\circ} \mathrm{C}$ with vigorous shaking. After every 24 -h period, the stationary phase culture was back-diluted in fresh LB broth to an $\mathrm{OD}_{600 \mathrm{~nm}}$ of 0.02 , for a total period of 7 days. Each day, the culture was sampled, serially diluted, and spread over LB agar and LB agar containing $0.5 \mu \mathrm{g} /$ $\mathrm{mL}$ erythromycin $/ 12.5 \mu \mathrm{g} / \mathrm{mL}$ lincomycin to obtain the CFU of total bacteria and erythromycin-resistant strain, respectively. Relative fitness was calculated by the formula ${ }^{54}$ :

$$
w=\frac{\log _{2}\left(\mathrm{CFU}_{\mathrm{erm}_{\mathrm{t}}^{\mathrm{R}}} / \mathrm{CFU}_{\mathrm{erm}}^{\mathrm{R}}\right)}{\log _{2}\left(\mathrm{CFU}_{\mathrm{erm}_{\mathrm{t}}^{\mathrm{s}}}^{\mathrm{s}} / \mathrm{CFU}_{\mathrm{erm}}^{\mathrm{s}}\right)}
$$

where $w$ means relative fitness of the erythromycin-resistant strain to the erythromycin-sensitive strain; $\mathrm{CFU}_{\mathrm{erm}_{0}^{\mathrm{R}}}$ and $\mathrm{CFU}_{\mathrm{erm}_{t}^{\mathrm{R}}}$ means the total CFU of erythromycin-resistant strain before and after competition, respectively; $\mathrm{CFU}_{\mathrm{erm}} \mathrm{s}$ and $\mathrm{CFU}_{\mathrm{erm}_{t}^{\mathrm{s}}}$ means the total CFU of erythromycin-sensitive strain before and after competition, respectively. The CFUs were adjusted according to the dilution factors of the back-dilutions.

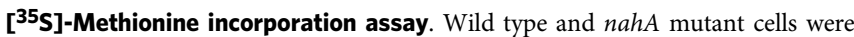
grown in defined S7 medium with glucose and 20 amino acids (concentrations listed in the "Plasmid and Strain Construction and Growth Conditions" section) to $\mathrm{OD}_{600 \mathrm{~nm}} \sim 0.3$. For amino acid starvation, cells were pelleted and resuspended in S7 glucose medium without amino acid. Alternatively, cells were treated with $0.5 \mathrm{mg} /$ $\mathrm{ml}$ arginine hydroxamate. Aliquots of $200 \mu \mathrm{L}$ culture were taken at indicated time points after treatment to label with $0.025 \mu \mathrm{Ci} / \mu \mathrm{L}\left[{ }^{35} \mathrm{~S}\right]$-Methionine for $5 \mathrm{~min}$, before adding $200 \mu \mathrm{L}$ ice-chilled $20 \%$ (w/v) trichloroacetic acid. Samples were chilled before filtration. Glass fiber filters (24 mm, GF6, Whatman) were prewetted with $3 \mathrm{~mL}$ ice-chilled $5 \%(\mathrm{w} / \mathrm{v})$ trichloroacetic acid and applied with samples. Filters were then washed three times by $3 \times 10 \mathrm{~mL}$ ice-chilled $5 \%(\mathrm{w} / \mathrm{v})$ trichloroacetic acid and dried by ethanol. Dried filters were put in scintillation vials, mixed with $5 \mathrm{~mL}$ scintillation fluid and then sent for scintillation count in the range of $2.0-18.6 \mathrm{eV}$. Counts per minutes (CPM) measured by scintillation counter, divided by the period of labeling and the $\mathrm{OD}_{600 \mathrm{~nm}}$ of the corresponding culture, were used as the representative of translation rate.

Growth recovery from nutrient downshift. Cells were grown in defined amino acid repleted medium (S7 medium with glucose, 20 amino acids, and $0.5 \mathrm{mM}$ IPTG) to mid $\log$ phase $\left(\mathrm{OD}_{600 \mathrm{~nm}} \sim 0.5\right)$, and then washed in S7 glucose without amino acid. Washed cultures were diluted into fresh medium with 20 amino acids and $0.5 \mathrm{mM}$ IPTG to $\mathrm{OD}_{600 \mathrm{~nm}}=0.01$ and the growth was monitored by a plate reader (Synergy 2, Biotek) at $37^{\circ} \mathrm{C}$ under vigorous shaking.

Reporting summary. Further information on research design is available in the Nature Research Reporting Summary linked to this article.

\section{Data availability}

Source data are provided with this paper.

Received: 5 May 2020; Accepted: 23 September 2020;

Published online: 23 October 2020

\section{References}

1. Cashel, M. in Escherichia coli and Salmonella Molecular and Cell Biology 1485-1496 (ASM, 1996).

2. Liu, K., Bittner, A. N. \& Wang, J. D. Diversity in (p)ppGpp metabolism and effectors. Curr. Opin. Microbiol. 24, 72-79 (2015).

3. Dalebroux, Z. D., Svensson, S. L., Gaynor, E. C. \& Swanson, M. S. ppGpp conjures bacterial virulence. Microbiol. Mol. Biol. Rev. 74, 171-199 (2010).

4. Ross, W., Vrentas, C. E., Sanchez-Vazquez, P., Gaal, T. \& Gourse, R. L. The magic spot: a ppGpp binding site on E. coli RNA polymerase responsible for regulation of transcription initiation. Mol. Cell 50, 420-429 (2013).
5. Ross, W. et al. ppGpp binding to a site at the RNAP-DksA interface accounts for its dramatic effects on transcription initiation during the stringent response. Mol. Cell 62, 811-823 (2016).

6. Molodtsov, V. et al. Allosteric effector ppGpp potentiates the inhibition of transcript initiation by DksA. Mol. Cell 69, 828-839 (2018).

7. Reddy, P. S., Raghavan, A. \& Chatterji, D. Evidence for a ppGpp-binding site on Escherichia coli RNA polymerase: proximity relationship with the rifampicin-binding domain. Mol. Microbiol. 15, 255-265 (1995).

8. Chatterii, D., Fujita, N. \& Ishihama, A. The mediator for stringent control, ppGpp, binds to the $\beta$-subunit of Escherichia coli RNA polymerase. Genes Cells 3, 279-287 (1998).

9. Wang, J. D., Sanders, G. M. \& Grossman, A. D. Nutritional control of elongation of DNA replication by (p)ppGpp. Cell 128, 865-875 (2007).

10. Rymer, R. U. et al. Binding mechanism of metal-NTP substrates and stringentresponse alarmones to bacterial DnaG-type primases. Structure 20, 1478-1489 (2012).

11. Maciag-Dorszyńska, M., Szalewska-Pałasz, A. \& Wegrzyn, G. Different effects of ppGpp on Escherichia coli DNA replication in vivo and in vitro. FEBS Open Bio 3, 161-164 (2013).

12. Kriel, A. et al. Direct regulation of GTP homeostasis by (p)ppGpp: a critical component of viability and stress resistance. Mol. Cell 48, 231-241 (2012).

13. Liu, K. et al. Molecular mechanism and evolution of guanylate kinase regulation by (p)ppGpp. Mol. Cell 57, 735-749 (2015).

14. Anderson, B. W. et al. Evolution of (p)ppGpp-HPRT regulation through diversification of an allosteric oligomeric interaction. Elife 8, e47534 (2019).

15. Wang, B. et al. Affinity-based capture and identification of protein effectors of the growth regulator ppGpp. Nat. Chem. Biol. 15, 141-150 (2019).

16. Feng, B. et al. Structural and functional insights into the mode of action of a universally conserved Obg GTPase. PLoS Biol. 12, e1001866 (2014).

17. DeLivron, M. A. \& Robinson, V. L. Salmonella enterica serovar Typhimurium BipA exhibits two distinct ribosome binding modes. J. Bacteriol. 190, 5944-5952 (2008)

18. Corrigan, R. M., Bellows, L. E., Wood, A. \& Gründling, A. ppGpp negatively impacts ribosome assembly affecting growth and antimicrobial tolerance in Gram-positive bacteria. Proc. Natl Acad. Sci. USA 113, E1710-E1719 (2016)

19. Buglino, J., Shen, V., Hakimian, P. \& Lima, C. D. Structural and biochemical analysis of the Obg GTP binding protein. Structure 10, 1581-1592 (2002).

20. Zhang, Y., Zborníková, E., Rejman, D. \& Gerdes, K. Novel (p)ppGpp binding and metabolizing proteins of Escherichia coli. MBio 9, e02188-17 (2018).

21. Mechold, U., Potrykus, K., Murphy, H., Murakami, K. S. \& Cashel, M. Differential regulation by ppGpp versus pppGpp in Escherichia coli. Nucleic Acids Res. 41, 6175-6189 (2013).

22. Gaca, A. O. et al. From (p)ppGpp to (pp)pGpp: characterization of regulatory effects of pGpp synthesized by the small alarmone synthetase of Enterococcus faecalis. J. Bacteriol. 197, 2908-2919 (2015).

23. Petchiappan, A., Naik, S. Y. \& Chatterii, D. RelZ-mediated stress response in Mycobacterium smegmatis: pGpp synthesis and its regulation. J. Bacteriol. 202, e00444-19 (2020).

24. Roelofs, K. G., Wang, J., Sintim, H. O. \& Lee, V. T. Differential radial capillary action of ligand assay for high-throughput detection of protein-metabolite interactions. Proc. Natl Acad. Sci. USA 108, 15528-15533 (2011).

25. Steinchen, W. et al. Catalytic mechanism and allosteric regulation of an oligomeric (p)ppGpp synthetase by an alarmone. Proc. Natl Acad. Sci. USA 112, 13348-13353 (2015)

26. Maki, H. \& Sekiguchi, M. MutT protein specifically hydrolyses a potent mutagenic substrate for DNA synthesis. Nature 355, 273-275 (1992).

27. Gropp, M., Eizenman, E., Glaser, G., Samarrai, W. \& Rudner, R. A relA ${ }^{\mathrm{S}}$ suppressor mutant allele of Bacillus subtilis which maps to relA and responds only to carbon limitation. Gene 140, 91-96 (1994).

28. Atkinson, G. C., Tenson, T. \& Hauryliuk, V. The RelA/SpoT homolog (RSH) superfamily: distribution and functional evolution of ppGpp synthetases and hydrolases across the tree of life. PLoS ONE 6, e23479 (2011).

29. Srivatsan, A. et al. High-precision, whole-genome sequencing of laboratory strains facilitates genetic studies. PLoS Genet. 4, 1-14 (2008).

30. Frindert, J. et al. YvcI from Bacillus subtilis has in vitro RNA pyrophosphohydrolase activity. J. Biol. Chem. 294, 19967-19977 (2019).

31. Maass, S. et al. Efficient, global-scale quantification of absolute protein amounts by integration of targeted mass spectrometry and two-dimensional gel-based proteomics. Anal. Chem. 83, 2677-2684 (2011).

32. Anderson, B. W., Hao, A., Satyshur, K. A., Keck, J. L. \& Wang, J. D. Molecular mechanism of regulation of the purine salvage enzyme XPRT by the alarmones pppGpp, ppGpp, and pGpp. J. Mol. Biol. 432, 4018-4126 (2020).

33. Krásný, L. \& Gourse, R. L. An alternative strategy for bacterial ribosome synthesis: Bacillus subtilis rRNA transcription regulation. EMBO J. 23, 4473-4483 (2004)

34. Kriel, A. et al. GTP dysregulation in Bacillus subtilis cells lacking (p)ppGpp results in phenotypic amino acid auxotrophy and failure to adapt to 
nutrient downshift and regulate biosynthesis genes. J. Bacteriol. 196, 189-201 (2014).

35. Liu, K. et al. Molecular mechanism and evolution of guanylate kinase regulation by (p)ppGpp. Mol. Cell 57, 735-749 (2015).

36. Zhang, Y. et al. HflX is a ribosome-splitting factor rescuing stalled ribosomes under stress conditions. Nat. Struct. Mol. Biol. 22, 906-913 (2015).

37. Basu, A. \& Yap, M. N. F. Disassembly of the Staphylococcus aureus hibernating $100 \mathrm{~S}$ ribosome by an evolutionarily conserved GTPase. Proc. Natl Acad. Sci. USA 114, E8165-E8173 (2017).

38. Hwang, J. \& Inouye, M. The tandem GTPase, Der, is essential for the biogenesis of $50 \mathrm{~S}$ ribosomal subunits in Escherichia coli. Mol. Microbiol. 61, 1660-1672 (2006).

39. Matsuo, Y. et al. The GTP-binding protein YlqF participates in the late step of $50 \mathrm{~S}$ ribosomal subunit assembly in Bacillus subtilis. J. Biol. Chem. 281 8110-8117 (2006).

40. Uicker, W. C., Schaefer, L. \& Britton, R. A. The essential GTPase RbgA (YlqF) is required for $50 \mathrm{~S}$ ribosome assembly in Bacillus subtilis. Mol. Microbiol. 59, 528-540 (2006).

41. Inoue, K., Alsina, J., Chen, J. \& Inouye, M. Suppression of defective ribosome assembly in a $r b f A$ deletion mutant by overexpression of Era, an essential GTPase in Escherichia coli. Mol. Microbiol. 48, 1005-1016 (2003).

42. Cabedo, H. The Escherichia coli trmE (mnmE) gene, involved in tRNA modification, codes for an evolutionarily conserved GTPase with unusual biochemical properties. EMBO J. 18, 7063-7076 (1999).

43. Trinquier, A. et al. tRNA maturation defects lead to inhibition of rRNA processing via synthesis of pppGpp. Mol. Cell 74, 1227-1238 (2019).

44. Nishino, T., Gallant, J., Shalit, P., Palmer, L. \& Wehr, T. Regulatory nucleotides involved in the Rel function of Bacillus subtilis. J. Bacteriol. 140, 671-679 (1979).

45. Lee, P. C., Bochner, B. R. \& Ames, B. N. AppppA, heat-shock stress, and cell oxidation. Proc. Natl Acad. Sci. USA 80, 7496-7500 (1983).

46. Ooga, T. et al. Degradation of ppGpp by nudix pyrophosphatase modulates the transition of growth phase in the bacterium Thermus thermophilus. J. Biol. Chem. 284, 15549-15556 (2009)

47. Denapoli, J., Tehranchi, A. K. \& Wang, J. D. Dose-dependent reduction of replication elongation rate by (p)ppGpp in Escherichia coli and Bacillus subtilis. Mol. Microbiol. 88, 93-104 (2013).

48. Lee, V. T. et al. A cyclic-di-GMP receptor required for bacterial exopolysaccharide production. Mol. Microbiol. 65, 1474-1484 (2007).

49. Burby, P. \& Simmons, L. CRISPR/Cas9 Editing of the Bacillus subtilis Genome. Bio-protocol 7, e2272 (2017).

50. Ben-Yehuda, S., Rudner, D. Z. \& Losick, R. RacA, a bacterial protein that anchors chromosomes to the cell poles. Science 299, 532-536 (2003).

51. Vasantha, N. \& Freese, E. Enzyme changes during Bacillus subtilis sporulation caused by deprivation of guanine nucleotides. J. Bacteriol. 144, 1119-1125 (1980).

52. Clasquin, M. F., Melamud, E. \& Rabinowitz, J. D. LC-MS data processing with MAVEN: A metabolomic analysis and visualization engine. Curr. Protoc. Bioinform. 37, 14.11.1-14.11.23 (2012).

53. Melamud, E., Vastag, L. \& Rabinowitz, J. D. Metabolomic analysis and visualization engine for LC-MS data. Anal. Chem. 82, 9818-9826 (2010).

54. Lenski, R. E. Experimental studies of pleiotropy and epistasis in escherichia coli. I. Variation in competitive fitness among mutants resistant to virus T4. Evolution 42, 425-432 (1988).
55. Konkol, M. A., Blair, K. M. \& Kearns, D. B. Plasmid-encoded ComI inhibits competence in the ancestral 3610 strain of Bacillus subtilis. J. Bacteriol. 195, 4085-4093 (2013)

\section{Acknowledgements}

We thank Mona Orr for technical help. We thank José Lemos for the gift of pGpp standard. This work is supported, in part, by an R35 GM127088 from NIGMS (to J.D.W.), a GRFP DGE-1256259 from NSF (to B.W.A.), 1715710 from NSF (to D.A.-N.), and R01AI110740 from NIAID and NIDDK (to V.T.L.).

\section{Author contributions}

B.W.A., V.T.L., and J.D.W. conceptualized the DRaCALA screen experiments. J.Y., B.W.A., A.T., H.T., V.T.L., and J.D.W. performed the library construction, J.Y., B.W.A., A.T., and H.T. performed the DRaCALA screen experiments. J.Y. and J.D.W. conceptualized the experiments on NahA. J.Y. performed most biochemical and physiological experiments. J.Y., D.M.S., and D.A.-N. designed LC-MS experiments. J.Y. and D.M.S. performed LC-MS experiments. J.Y. analyzed data for this work. J.Y. and J.D.W. wrote the manuscript. All authors edited the manuscript. V.T.L. and J.D.W. supervised the study. Funding was acquired by J.D.W. and V.T.L.

\section{Competing interests}

The authors declare no competing interests.

\section{Additional information}

Supplementary information is available for this paper at https://doi.org/10.1038/s41467020-19166-1.

Correspondence and requests for materials should be addressed to V.T.L.; or J.D.W.

Peer review information Nature Communications thanks Michael Cashel, Yong Zhang and the other, anonymous, reviewer for their contribution to the peer review of this work. Peer reviewer reports are available.

Reprints and permission information is available at http://www.nature.com/reprints

Publisher's note Springer Nature remains neutral with regard to jurisdictional claims in published maps and institutional affiliations.

(c) (i) Open Access This article is licensed under a Creative Commons Attribution 4.0 International License, which permits use, sharing, adaptation, distribution and reproduction in any medium or format, as long as you give appropriate credit to the original author(s) and the source, provide a link to the Creative Commons license, and indicate if changes were made. The images or other third party material in this article are included in the article's Creative Commons license, unless indicated otherwise in a credit line to the material. If material is not included in the article's Creative Commons license and your intended use is not permitted by statutory regulation or exceeds the permitted use, you will need to obtain permission directly from the copyright holder. To view a copy of this license, visit http://creativecommons.org/ licenses/by/4.0/.

(c) The Author(s) 2020, corrected publication 2021 